\title{
Perioperative Treatment in Gastric Cancer-Developments in Patient Selection
}

\author{
Manuela Machado*, Helena Magalhães, Mário Fontes-Sousa \\ Medical Oncology Department, Portuguese Institute of Oncology of Porto (IPO Porto), Porto, Portugal \\ Email: *m.machado.fn@gmail.com
}

How to cite this paper: Machado, M., Magalhães, H. and Fontes-Sousa, M. (2018) Perioperative Treatment in Gastric CancerDevelopments in Patient Selection. Journal of Cancer Therapy, 9, 101-105. https://doi.org/10.4236/jct.2018.92011

Received: December 21, 2017

Accepted: January 30, 2018

Published: February 2, 2018

Copyright $\odot 2018$ by authors and Scientific Research Publishing Inc. This work is licensed under the Creative Commons Attribution International License (CC BY 4.0).

http://creativecommons.org/licenses/by/4.0/

(c) (i) Open Access

\begin{abstract}
Gastric cancer (GC) is a major public health issue. It is considered the $5^{\text {th }}$ most common cancer diagnosed worldwide and it is one of the main causes of malignant disease-associated morbidity and mortality. The cornerstone of curative treatment is still surgery, and since the rate of relapse is high, a multidisciplinary approach is warranted in most developed countries. And while there have been recent developments in the perioperative scenario namely the FLOT regimen, little has advanced considering patient selection. We have reviewed the major trials in this setting and provide some insights from recently reported microsatellite instability (MSI) in a subgroup analysis in the MAGIC trial patients that seem to suggest an opportunity to patient selection. Furthermore, GC subtyping may prove helpful selecting candidates to immunotherapy or even multimodal therapy in the future. As the paradigm is moving towards a precision oncology model, GC patient selection remains one the biggest challenges in oncology but seems closer to clinical practice reality as new developments are being reported.
\end{abstract}

\section{Keywords}

Gastric Cancer, Perioperative Chemotherapy, Molecular Classification of Gastric Cancer, MSI

\section{Introduction}

Gastric cancer (GC) represents the $5^{\text {th }}$ most common cancer diagnosed worldwide, being one of the main causes of morbidity and mortality due to malignant disease. The incidence of GC varies geographically, being particularly higher in the Asiatic population compared to the Western countries. In Europe, almost 107,000 deaths occurred in 2012 [1] [2]. Currently, the only curative treatment for GC is surgery, with survival rates at 5 years of $70 \%-75 \%$ in stage I and $35 \%$ 
in stage II and III for completely resected GC, with several patients experiencing recurrence during follow up [3]. Those disappointing numbers lead to different strategies that should be discussed in a multidisciplinary scenario: neoadjuvant/perioperative and adjuvant therapy, all currently accepted as possible and valid strategies.

As the field is moving towards a precision oncology paradigm in clinical practice, patient selection becomes increasingly important-how to identify the patients that would benefit the most, while sparing them unnecessary toxicities. On the other hand, being Medical Oncologists who treat gastric patients in our routine clinical practice, we understand the need for better treatments, as relapse is predominantly a synonym of a dire prognosis. The authors will review some of the most relevant data and discuss its implications.

\section{Materials and Methods}

We identified the positive phase III trials in the perioperative setting in gastric or gastro-esophageal junction using only chemotherapy, not radiotherapy or chemoradiotherapy treatments. In parallel, we conducted a search for the latest and most credible evidence regarding molecular profiling for patient selection in perioperative gastric cancer patients.

\section{Discussion}

The evidence for perioperative chemotherapy in GC in Europe was based in two published studies: the MAGIC trial, in 2006, FNLCC ACCORD 07/FFCD 9703 trial, 5 years later [4] [5].

The randomized phase III MAGIC trial, conducted in the United Kingdom, included patients with resectable adenocarcinoma of the stomach, gastroesophageal junction (GEJ), and lower esophagus: 250 patients allocated to the perioperative treatment arm versus 253 patients allocated to the surgery alone arm. The chemotherapy regimen consisted of 3 preoperative and 3 postoperative cycles of epirubicin, cisplatin and 5-fluorouracil for 21 days cycle. The perioperative chemotherapy arm showed improved results: 5-year survival rates (36.3\% versus $23 \%$ ) with a likelihood of $\mathrm{OS}(\mathrm{HR}, 0.75 ; 95 \mathrm{CI}, 0.60$ to 0.93 ; $\mathrm{P}=0.009$ ).

The FNLCC ACCORD 07/FFCD 9703 phase III trial included 224 patients with adenocarcinoma (75\% from gastroesophageal junction or lower esophagus and only $25 \%$ from stomach). The trial had two arms: perioperative chemotherapy plus surgery versus surgery alone, with a recommended D2 lymph node dissection. The perioperative chemotherapy schedule included cisplatin and fluorouracil ( 2 - 3 preoperative and 3 - 4 postoperative). The perioperative chemotherapy arm had significantly better curative resection rate $(84 \% \mathrm{v} 73 \% ; \mathrm{P}=$ 0.04 ), improved OS (5-year rate $38 \%$ v $24 \%$; hazard ratio [HR] for death: 0.69; $95 \% \mathrm{CI}, 0.50$ to $0.95 ; \mathrm{P}=0.02$ ) and disease-free survival (DFS), with 5 years DFS rates of $34 \%$ in the combined arm (vs $19 \%$ in the surgery alone; HR, $0.65 ; 95 \%$ CI, 0.48 to $0.89 ; \mathrm{P}=0.003$ ). 
In June 2017, the results of the FLOT4-AIO trial were presented as abstract at the American Society of Clinic Oncology (ASCO) annual meeting and since then this regimen has been generally accepted as the promising new standard of care for perioperative chemotherapy in GC [6] [7] [8] [9], although its final publication is eagerly awaited. This phase II/III trial, in resectable GC $\geq \mathrm{cT} 2$ and/or $\mathrm{cN}+$ patients, tested perioperative chemotherapy with docetaxel, oxaliplatin, and fluorouracil/leucovorin (FLOT) - 4 cycles preoperative plus 4 cycles postoperativeversus the standard after the MAGIC trial, epirubicin, cisplatin and fluorouracil (ECF) or epirubicin, cisplatin and capecitabine (ECX)-3 cycles preoperative plus 3 cycles postoperative. With a median follow-up of 43 months, the authors reported that FLOT improved both OS (mOS, 35 mon with ECX/ECF vs. 50 mon with FLOT; HR 0.77 [0.63 - 0.94]; $\mathrm{p}=0.012$ ) and PFS (mPFS, 18 mon with ECX/ECF vs. 30 mon with FLOT; HR 0.75 [0.62 - 0.91]; $p=0.004$ ). Considering the toxicities, there was more G3/4 nausea and vomiting with ECF/ECX and more G3/4 neutropenia with FLOT (51\% with FLOT versus 39\% with ECF/ECX).

Additionally, one of the major pitfalls considering GC staging is accurate nodal disease assessment - that is generally considered to be under evaluated by standard imaging, such as computed tomography (CT) scan. The sensitivity for CT scan in diagnosing malignant lymph node disease is variable [10], and in our clinical practice experience it can be even lower than published in the literature (35\% vs $60 \%$ - 90\%) [11]. Still today, a global consensus on malignant lymph node disease diagnostic criteria is not available [10], so we believe that a better understanding of the role of the different imaging modalities is necessary for better patient selection.

In February 2017, an exploratory post hoc analysis from the MAGIC trial was published [12]. The goal was to evaluate the proportion of patients with high MSI (MSI-H) or mismatch repair deficiency (MMRD) in the 2 cohorts, and if these biomarkers had prognostic effect on survival. Of the 456 patients who underwent surgery in the MAGIC trial, MSI results were available for 303 patients, and only 20 had MSI-H, all located in primary gastric tumors. Compared to MSS (stable) or MSI-L (low) tumors, patients with MSI-H were more frequent in female, with older median age and intestinal subtype, but none of these differences were statistically significant. Patients treated with surgery alone who had MSI-H or MMRD had a median OS that was not reached (95\% CI, 11.5 months to not reached) versus 20.5 months (95\% CI, 16.7 - 27.8 months; HR 0.42; 95\% CI, 0.15 $1.15 ; P=0.09$ ) among those who had neither MSI-H nor MMRD. In contrast, patients treated with chemotherapy plus surgery who had either high MSI or MMRD had a mOS of 9.6 months (95\% CI, $0.1-22.5$ months) compared with the mOS among those who were neither MSI-H nor MMRD of 19.5 months (95\% CI, 15.4 - 35.2 months; hazard ratio, 2.18; 95\% CI, 1.08 - 4.42; $P=0.03$ ). Despite the limitations of this exploratory analysis, the authors concluded that MMRD and MSI-H were correlated with a positive prognostic effect in patients treated with surgery alone and a negative prognostic effect in the perioperative group, thus suggesting a potential development in patient selection. Although 
these results seem promising, we believe that due to the low patient numbers (around 20) the evidence may not be strong enough to change clinical practice yet. Furthermore, these results may not be extrapolated to predict "chemoresistance" in the general population, since that in the MAGIC trial the regimen included anthracycline but now the standard regimen FLOT does not. It would be useful to explore these principles in the FLOT4-AIO trial subpopulations in order to understand if the concept of "chemoresistance" is regimen-associated or not.

A major breakthrough in understanding GC heterogeneity was the Cancer Genome Atlas research network (TCGA) publication in 2014, that suggested 4 subtypes of gastric tumours, based on their molecular signature: Epstein Barr virus (EBV) positive, microsatellite unstable (MSI), genomically stable (GS) and chromosomal instability (CIN) [13]. Of these, the EBV positive and MSI subtypes have a higher mutational burden and it may play a role in immune response, rendering them as potential candidates for "better responders" to immunotherapy drugs [14]. For instance, the role of perioperative Pembrolizumab, an anti-PD-1 drug, is already ongoing (ClinicalTrials.gov Identifier: NCT02918162).

\section{Conclusions and Future Directions}

As tumor heterogeneity in GC is increasingly recognized and molecular subtyping is being further understood, the field is evolving at a fast pace and new standards of care may emerge in the near future. One of the latest practice changing novelties was the perioperative regimen FLOT in resectable GC that improved OS and PFS when compared to the MAGIC trial regimen. However, insights regarding MSI in subgroup analysis in the MAGIC trial patients seem to suggest an opportunity to patient selection. Furthermore, advancing GC subtyping may prove helpful selecting candidates to immunotherapy-still experimental in this setting, but demonstrated in the metastatic patients-or even multimodal therapy selection. Standardizing imaging criteria for lymph node disease assessment is a fundamental need for accurate staging. As the paradigm is moving towards a precision oncology model, GC patient selection remains one the biggest challenges in oncology today.

\section{References}

[1] Arnold, M., Karim-Kos, H.E., Coebergh, J.W., et al. (2015) Recent Trends in Incidence of Five Common Cancers in 26 European Countries since 1988: Analysis of the European Cancer Observatory. European Journal of Cancer, 51, 1164-1187. https://doi.org/10.1016/j.ejca.2013.09.002

[2] Ferlay, J., Soerjomataram, I., Dikshit, R., et al. (2015) Cancer Incidence and Mortality Worldwide: Sources, Methods and Major Patterns in GLOBOCAN 2012. International Journal of Cancer, 136, E359-E386. https://doi.org/10.1002/ijc.29210

[3] Data from the SEER 1973-2005 Public Use File Diagnosed in Years 1991 to 2000, published in UpToDate.com.

[4] Cunningham, D., Allum, W.H., Stenning, S.P., et al. (2006) Perioperative Chemotherapy versus Surgery Alone for Resectable Gastroesophageal Cancer. New England Journal of Medicine, 355, 11-20. https://doi.org/10.1056/NEJMoa055531 
[5] Ychou, M., Boige, V., Pignon, J.-P., et al. (2011) Perioperative Chemotherapy Compared with Surgery Alone for Resectable Gastroesophageal Adenocarcinoma: An FNCLCC and FFCD Multicenter Phase III Trial. Journal of Clinical Oncology, 29, 1715-1721. https://doi.org/10.1200/JCO.2010.33.0597

[6] Al-Batran, S.-E, Hartmann, J.T., et al. (2008) Biweekly Fluorouracil, Leucoverin, Oaxaliplatin and Docetaxel (FLOT) for Patients with Metastatic Adenocarcinoma of Stomach or Esophagogastric Junction: A Phase II Trial of Arbeitsgemeinschaft Internistische Onckologie. Annals of Oncology, 19, 1882-1887.

https://doi.org/10.1093/annonc/mdn403

[7] Lorenzen, S., Thus-Patience, P., et al. (2013) Impact of Pathologic Complete Response on Disease Free Survival in Patients with Esophagogastric Adenocarcinoma Receiving Preoperative Docetaxel-Based Chemotherapy. Annals of Oncology, 24, 2068-2073. https://doi.org/10.1093/annonc/mdt141

[8] Al Batran, S.-E., Hofheinz, R.D., et al. (2016) Histopathological Regression after Neoadjuvant Docetaxel, Oxaliplatin, Fluorouracil or Capecitabine in Patients with Resectable Gastric or Gastro-Oesophageal Junction Adenocarcinoma (FLOT4-AIO): Results from the Phase 2 Part of a Multicentre, Open-Label, Randomised Phase 2/3 Trial. Lancet Oncology, 17, 1697-1708. https://doi.org/10.1016/S1470-2045(16)30531-9

[9] Al Batran, S.E. (2017) Perioperative Chemotherapy with Docetaxel, Oxaliplatin and Fluorouracil/Leucoverin (FLOT) versus Epirrubicin, Cisplatin and Fluorouracil or Capecitabine (ECF/ECX) for Ressectable Gastric or Gastroesophageal Junction (GEJ) Adenocarcinoma (FLOT4): A Multicenter, Randomized Phase 3 Trial. Oral Presentation, presented at 2017 ASCO Annual Meeting. https://doi.org/10.1200/JCO.2017.35.15_suppl.4004

[10] Smyth, E.C., Verheij, M., Allum, W., Cunningham, D., Cervantes, A. and Arnold, D. (2016) Gastric Cancer: ESMO Clinical Practice Guidelines for Diagnosis, Treatment and Follow-Up. Annals of Oncology, 27, v38-v49. https://doi.org/10.1093/annonc/mdw350

[11] Fontes e Sousa, M., Magalhães, H., Araújo, R., et al. (2017) The Role of Computed Tomography for Node Staging in Gastric Cancer: A Center's Retrospective Analysis. Annals of Oncology, 28, Issue suppl. 3. https://doi.org/10.1093/annonc/mdx261.096

[12] Smyth, E., Wotherspoon, A., et al. (2017) Mismatch Repair Deficiency, Microsatellite Instability, and Survival: An Exploratory Analysis of the Medical Research Council Adjuvant Gastric Infusional Chemotherapy (MAGIC) Trial. JAMA Oncology, 3, 1197-1203. https://doi.org/10.1001/jamaoncol.2016.6762

[13] Bass, A.J., Thorsson, V., Shmulevich, I., et al. (2014) Comprehensive Molecular Characterization of Gastric Adenocarcinoma. Nature, 513, 202-209. https://doi.org/10.1038/nature 13480

[14] Garattini, S., Basile, D., Cattaneo, M., et al. (2017) Molecular Classifications of Gastric Cancers: Novel Insights and Possible Future Applications. World Journal of Gastrointestinal Oncology, 9, 194-208. https://doi.org/10.4251/wjgo.v9.i5.194 